\title{
Expresión del receptor de andrógenos relativa al receptor de estrógenos (AR/ER) y su correlación con una señal génica de proliferación en cáncer de seno ER-positivo
}

\author{
Androgen receptor expression relative to estrogen receptor expresion (AR/ \\ $E R$ ) and relationship with a gene proliferation signature in ER-positive \\ breast cancer
}

Rangel $N^{1,2}$, Aristizábal AF $F^{3}$, Rondón $M^{1}$.

${ }^{1} \mathrm{PhD}$. Grupo de Investigación en Ciencias Biomédicas (GICB), Escuela de Ciencias Biológicas, Facultad de Ciencias, Universidad Pedagógica y Tecnológica de Colombia (UPTC), Tunja, Colombia. ${ }^{2}$ Departamento de Ciencias Médicas, Unidad de Patología, Hospital Città della Salute e della Scienza di Torino, Turín, Italia.

${ }^{3} \mathrm{PhD}$. Departamento de Nutrición y Bioquímica, Facultad de Ciencias, Pontificia Universidad Javeriana, Bogotá, Colombia.

Autor de correspondencia: Nelson Rangel

Correo electrónico: nelson.rangel01@uptc.edu.co

Fecha de recepción: 27/11/2018

Fecha de aceptación: 30/01/2019

\section{Resumen}

Objetivo: previamente se reportó que tumores de seno con proporción receptor de andrógenos/receptor de estrógenos $\geq 2(A R / E R \geq 2)$ se asocian con peores pronósticos. De acuerdo con lo anterior, el objetivo del presente estudio fue evaluar la relación entre la proporción AR/ER y una señal génica de proliferación celular en cáncer de seno (CS; también conocido como cáncer de mama) ER positivo (ER+).

Métodos: se obtuvieron 1093 muestras de CS primario con datos de expresión génica de la base de datos pública NCI Genomic Data Commons. Se ejecutaron análisis bioinformáticos para establecer niveles de expresión génica de AR y ER, así como de los marcadores de proliferación AURKb, BUB1B, BUB1, CDK1 y CHEK1. Adicionalmente, se definieron subtipos moleculares de CS utilizando el bioclasificador PAM50.

Resultados: 835 casos se reportaron como ER+. Entre estos, 58 tumores $(7 \%)$ presentaron mayores niveles de expresión de AR respecto a ER (AR/ER $\geq 2)$. En dichos casos se observaron niveles significativamente más altos de proliferación con respecto a tumores, con proporción AR/ER $<2(p=0,02), y$ preferencialmente se clasificaron dentro de los subtipos moleculares luminal B y HER2 enriquecido (76,5\%).

Conclusión: nuestros resultados confirman reportes previos y sugieren que los casos de CS con proporción $A R / E R \geq 2$ se asocian con tumores que tienen un comportamiento biológico más agresivo. Asimismo, se refuerza la idea de utilizar inhibidores de AR para el tratamiento de pacientes con CS ER+ que presentan altos niveles de expresión de este tipo de cáncer.

Palabras clave: cáncer de seno, receptor de andrógenos, receptor de estrógenos, proliferación celular, subtipos moleculares.

\section{Abstract}

Purpose: Previously have been reported that breast cancers $(B C)$ with high expression of AR relative to estrogen receptor $(A R / E R \geq 2)$ are associated with worse prognosis. The aim of this study was to evaluate the relationship between the AR/ER ratio and a gene proliferation signature in ER - positive (ER+) tumors.

Methods: 1093 BC samples with gene expression data were obtained from NCI Genomic Data Commons. Bioinformatic analysis were performed to establish gene expression levels of $A R, E R$ and also levels of cellular proliferation markers $A U R K b$, $B U B 1 B, B U B 1, C D K 1$ and CHEK1. Furthermore, molecular subtypes were defined based on the PAM50 bioclassifier.

Results: 835 cases were reported as ER+ and, from them, 58 tumors (7\%) showed higher level of AR respect to ER (AR) $E R \geq 2$ ). These last cases had significantly higher levels of cellular proliferation markers, compared with $A R / E R<2$ ratio cases ( $p=0.02)$ and were mainly classified as luminal B and HER2enriched molecular subtypes (76.5\%). 
Conclusions: our results confirm previous reports and suggest that $B C$ cases with ratio $A R / E R \geq 2$ are associated with tumors having more aggressive biological behavior. Moreover, it reinforces the insight of use of AR-inhibitors for treatment of ER+ BC patients which have higher AR expression levels.

Keywords: Breast Neoplasms, Receptors, Androgen; Receptors, Estrogen, Cell Proliferation, Gene Expression Profiling, Molecular typing.

\section{Introducción}

El cáncer de seno (CS; o cáncer de mama) es una enfermedad ampliamente reconocida como hormono-dependiente, ya que receptores de estrógenos (ER) y progesterona (PR) son expresados en la gran mayoría de tumores de seno $(\sim 75 \%)$ y ambos han mostrado utilidad significativa como marcadores de pronóstico y predicción al tratamiento ${ }^{(1)}$. Sin embargo, el significado clínico y biológico en CS de otro receptor hormonal, tal como lo es el receptor de andrógenos (AR), no se ha definido claramente. El AR es un factor de transcripción citoplasmático dependiente de ligando (testosterona o dihidrotestosterona) y su positividad se ha detectado en más del $61 \%$ de las lesiones primarias y metastásicas de $\mathrm{CS}^{(2,3)}$. Alrededor del 75\% de los tumores de seno ER-positivos (ER+) es también AR-positivo (AR+). Muchos estudios han mostrado que la expresión de $\mathrm{AR}$ en tumores luminales $(\mathrm{ER}+)$ se considera un factor independiente asociado con características de buen pronóstico (bajo grado, tamaño tumoral pequeño, bajo índice proliferativo y supervivencia libre de enfermedad más larga) $)^{(4-7)}$. Por otro lado, más del 31\% de los tumores de seno ER negativo (ER-) se reporta como $\mathrm{AR}+$, pero el valor pronóstico de este marcador en el subgrupo de CS ER- no es claro, ya que investigaciones originales y metaanálisis muestran resultados contradictorios ${ }^{(2,3,7-14)}$.

Patrones de expresión similares entre AR y ER son evidentes en tejido de seno normal y a nivel celular ambos colocalizan, lo que indica que pueden ocurrir interacciones entre las vías de señalización de estos dos receptores ${ }^{(15)}$. De hecho, estudios in vitro muestran que la señalización de AR inhibe la proliferación mediada por ER en células luminales de CS (MCF7 - ER+) cuando esta es inducida por estrógenos ${ }^{(16-18)}$. Este efecto inhibidor de AR parece ser regulado por diferentes mecanismos, pero el más importante incluye la habilidad que tiene AR para competir con ER por los elementos de respuesta a estrógenos (ERE), lo que bloquea la transcripción de genes blanco dependientes de ER implicados en la proliferación celular ${ }^{(19)}$. En línea con estas observaciones, diferentes autores han reportado que incrementar la expresión de AR relativa a ER conllevaría a una mayor inhibición de la función protumorogénica de $\mathrm{ER}^{(15,20)}$

Por el contrario, a pesar del efecto aparentemente inhibidor de AR en células ER+, algunos autores han reportado proliferación incrementada de la línea celular MCF7 cuando se estimula la señalización de $\mathrm{AR}^{(21,22)}$. Además, varios estudios han demostrado extensamente que AR puede promover proliferación de células de CS ER-, particularmente en el subgrupo de CS triple negativo (ER-/PgR-/HER2-), conocido como apocrino o subtipo molecular LAR (luminal AR) ${ }^{(23,24)}$. Asimismo, y de acuerdo con lo anterior, recientes investigaciones establecen que casos de CS ER+ con altos niveles de AR respecto a ER $(\mathrm{AR} / \mathrm{ER} \geq 2)$ tienen tumores asociados con características clínicas y biológicas más agresivas, son resistentes al tamoxifeno y presentan períodos de supervivencia libre de la enfermedad más cortos; todas estas características relacionadas con pobres pronósticos en $\mathrm{CS}^{(25,26)}$.

La evidencia anterior sugiere que AR podría tener papeles duales en el CS relacionados con la proliferación celular, los cuales son dependientes del estado de ER (ER+ vs. ER-). Sin embargo, al considerar que niveles de positividad variable de ER son normalmente reportados en tumores luminales (ER+), en el presente estudio evaluamos si cambios en los niveles de expresión de AR respecto a ER (proporción AR/ER) pueden ser indicativos de variaciones en los niveles de proliferación celular en el subgrupo de tumores $\mathrm{ER}+{ }^{(27)}$. Para ello analizamos los niveles de ácido ribonucleico mensajero (ARNm) tanto del gen AR como del gen ESR1 (ER) en una cohorte de pacientes con CS obtenidos de bases de datos públicas y relacionamos la proporción AR/ER observada en estos casos con el perfil de expresión génica de un grupo de marcadores de proliferación celular (señal de proliferación celular [SPC]).

\section{Materiales y métodos}

\section{Casos y cálculo de la proporción AR/ER}

Una cohorte de 1093 muestras de CS primario, con información clinicopatológica y datos de expresión génica (RNA sequencing) se obtuvo de la base de datos publica NCI Genomic Data Commons (GDC) (https://gdc.cancer.gov). La expresión génica de los ARNm analizados se obtuvo tras un nivel 3 de análisis con el método de RSEM (RNA Seq by Expectation-Maximization), normalizado con el cuartil superior, para así mantener estandarización no solo entre las diferentes plataformas, sino también entre genes de referencia (genes normalizadores). Con el número de conteos normalizados se calculó la proporción AR/ER al dividir el nivel de expresión de AR con el nivel de expresión de ESR1. Teniendo en cuenta publicaciones previas se dividieron casos de CS ER+ en dos subgrupos para establecer comparaciones en análisis ulteriores (casos con proporción AR/ER<2 vs. casos con proporción AR/ER $\geq 2)^{(25,26)}$.

\section{Evaluación de la señal de proliferación celular}

Utilizando la misma estrategia de cuantificación génica se evaluó de forma individual y grupal los niveles de expresión (ARNm) de los genes AURKb, BUB1B, BUB1, CDK1 y CHEK1, previamente reportados como marcadores de proliferación celular $^{(28)}$. La evaluación grupal, tomada como el promedio de expresión de los 5 genes estudiados (señal de proliferación 
celular - SPC), se empleó para establecer asociaciones potenciales con los subgrupos de CS definidos por la proporción AR/ ER. Adicionalmente, subtipos moleculares de CS se definieron basados en el bioclasificador PAM50, descrito inicialmente por Parker y colaboradores utilizando el paquete para R, TCGABiolinks v. 2.7.1 ${ }^{(29,30)}$.

\section{Análisis estadísticos}

Se determinaron diferencias estadísticamente significativas tanto para variables categóricas como continuas entre los grupos de estudio, aplicando la prueba exacta de Fisher, $\chi^{2}$ y la prueba t de Student, dependiendo del caso. Se ejecutaron pruebas para medias y mediadas (análisis de varianza [ANOVA]) para establecer diferencias en los niveles de expresión génica entre grupos, complementados con la prueba de Dunnet y Tuckey HSD post-hoc para el análisis de tres o más grupos. Valores de $p<0,05$ se consideraron estadísticamente significativos. Todos los análisis se realizaron usando R: lenguaje y ambiente para cálculos estadísticos ${ }^{(31)}$.

\section{Resultados}

Del total de casos estudiados, 76,4\% (835) se reportó como ER+ por inmunohistoquímica en la base de datos pública GDC (tabla 1). Al evaluar los niveles de expresión de los receptores hormonales y calcular la proporción AR/ER dentro del grupo de casos ER+, 58 pacientes (7\%) presentaron niveles de AR/ $E R \geq 2$. Las principales características clinicopatológicas de los 835 casos ER+, estratificados por la proporción AR/ER, se informan en la tabla 1.

Casos con proporción AR/ER $\geq 2$ mostraron de manera significativa $(p<0,01)$ ser negativos para $\mathrm{PgR}$ y positivos para HER2, características normalmente asociadas con tumores más agresivos. Asimismo, el análisis de expresión individual y agrupado (SPC) de los genes de proliferación celular (AURKb, BUB1B, BUB1, CDK1 y CHEK1) mostró que los niveles de expresión de estos marcadores aumentan significativamente en el grupo de casos ER+, que expresan AR en mayor cantidad respecto a $E R$ (casos $A R / E R \geq 2$ ) (figura 1).

Tabla 1. Características clinicopatológicas de casos de CS estudiados

\begin{tabular}{|c|c|c|c|c|c|c|}
\hline \multirow[t]{2}{*}{ Variables } & \multirow[t]{2}{*}{ Todos (\%) } & \multicolumn{2}{|c|}{ ER+ } & \multirow{2}{*}{$\begin{array}{l}\text { Valor } \\
\text { de } p\end{array}$} & \multirow[t]{2}{*}{ ER- (\%) } & \multirow{2}{*}{$\begin{array}{c}\text { Valor } \\
\text { de } p\end{array}$} \\
\hline & & AR/ER $<2(\%)$ & $A R / E R \geq 2(\%)$ & & & \\
\hline Número total de casos & 1093 & $777(71,1)$ & $58(5,3)$ & & $258(23,6)$ & \\
\hline Edad (media) & 58,4 & 59,8 & 52,7 & $<0,001^{a}$ & 55,6 & $<0,001^{b}$ \\
\hline \multicolumn{7}{|l|}{ Estadio $^{\dagger}$} \\
\hline I & $181(17,12)$ & $136(18,2)$ & $9(16,1)$ & $0,329^{c}$ & $36(14,3)$ & $0,162^{c}$ \\
\hline II & $611(57,81)$ & $412(55)$ & $37(66,1)$ & & $162(64,3)$ & \\
\hline III & $246(23,27)$ & $186(24,8)$ & $10(17,9)$ & & $50(19,8)$ & \\
\hline IV & $19(1,8)$ & $15(2,0)$ & $0(0)$ & & $4(1,6)$ & \\
\hline \multicolumn{7}{|l|}{ Grado* } \\
\hline 1 & $181(16,93)$ & $136(17,9)$ & $9(16,1)$ & $0,242^{c}$ & $36(14,3)$ & $0,078^{c}$ \\
\hline 2 & $619(57,9)$ & $420(55,2)$ & $37(66,1)$ & & $162(64,3)$ & \\
\hline 3 & $269(25,16)$ & $205(26,9)$ & $10(17,9)$ & & $54(21,4)$ & \\
\hline \multicolumn{7}{|l|}{ Tamaño** } \\
\hline$<20 \mathrm{~mm}$ & $279(25,6)$ & $207(26,7)$ & $16(27,6)$ & $0,878^{d}$ & $56(21,8)$ & $0,275^{c}$ \\
\hline$\geq 20 \mathrm{~mm}$ & $811(74,4)$ & $568(73,3)$ & $42(72,4)$ & & $201(78,2)$ & \\
\hline \multicolumn{7}{|l|}{ Linfonodos $* * *$} \\
\hline 0 & $515(47,99)$ & $347(45,6)$ & $21(37,5)$ & $0,460^{c}$ & $147(57,4)$ & $0,009^{c}$ \\
\hline $1-3$ & $481(44,82)$ & $357(46,9)$ & $31(55,4)$ & & $93(36,3)$ & \\
\hline$>3$ & $77(7,18)$ & $57(7,5)$ & $4(7,1)$ & & $16(6,2)$ & \\
\hline \multicolumn{7}{|l|}{$\mathrm{PgR}^{+}$} \\
\hline Positivo & $699(67)$ & $640(86,7)$ & $35(70)$ & $0,01^{d}$ & $23(9,1)$ & $<0,001^{c}$ \\
\hline Negativo & $344(33)$ & $98(13,3)$ & $15(30)$ & & $229(90,9)$ & \\
\hline
\end{tabular}


Tabla 1. Características clinicopatológicas de casos de CS estudiados (continuación)

\begin{tabular}{|c|c|c|c|c|c|c|}
\hline \multirow[t]{2}{*}{ Variables } & \multirow[t]{2}{*}{ Todos (\%) } & \multicolumn{2}{|c|}{ ER+ } & \multirow{2}{*}{$\begin{array}{l}\text { Valor } \\
\text { de } p\end{array}$} & \multirow[t]{2}{*}{ ER- (\%) } & \multirow{2}{*}{$\begin{array}{l}\text { Valor } \\
\text { de } p\end{array}$} \\
\hline & & AR/ER <2 (\%) & $A R / E R \geq 2(\%)$ & & & \\
\hline \multicolumn{7}{|l|}{ HER2 $^{\ddagger}$} \\
\hline Positivo & $164(22,5)$ & $100(19,8)$ & $19(47,5)$ & $<0,001^{d}$ & $164(22,5)$ & $<0,001^{c}$ \\
\hline Negativo & $564(77,59)$ & $406(80,2)$ & $21(52,5)$ & & $133(74,7)$ & \\
\hline
\end{tabular}

HER2: receptor 2 del factor de crecimiento epidérmico humano; PgR: receptor de progesterona.

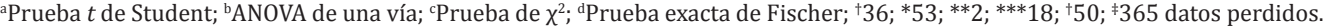

Los casos se agrupan de acuerdo con el estado de ER: casos $\mathrm{ER}+$, a su vez, se subdividieron teniendo en cuenta diferencias en la proporción $\mathrm{AR} / \mathrm{ER}(<2$ vs. $\geq 2)$.

Figura 1. Expresión de genes de proliferación celular en casos de CS. Niveles significativamente más altos se observaron en casos de CS con proporción $\mathrm{AR} / \mathrm{ER} \geq 2$, tanto en el análisis de expresión de genes individuales (AURKb, BUB1B, BUB1, CDK1 y CHEK1) como en el análisis grupal (SPC) de dichos genes. Niveles significativamente más altos de todos los genes también se observaron en casos ER
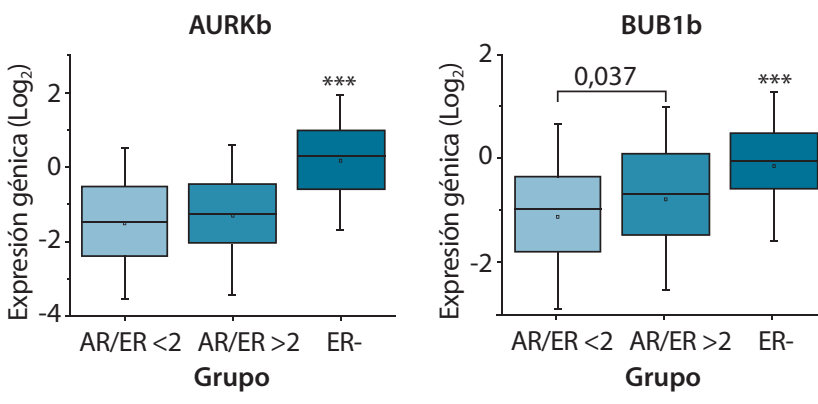

BUB1
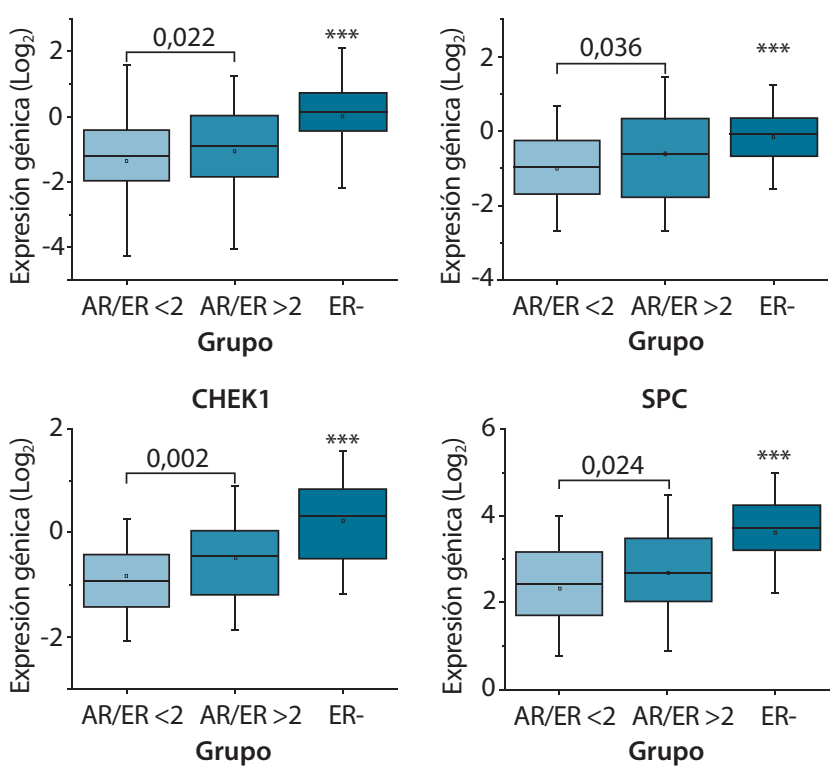

ER: casos de receptor de estrógenos negativo; $\log _{2}$ : logaritmo del nivel de expresión génica.

Valores de $p$ : prueba de comparaciones múltiples de Dunnet y Tuckey. $* * *<0,001$.
Adicionalmente, para tratar de categorizar mejor los casos de CS ER+ obtenidos de las bases de datos públicas se lograron definir subtipos moleculares en 1043 de los casos usando el bioclasificador PAM50 y se agruparon siguiendo diferencias en la proporción AR/ER ( $<2 v s . \geq 2)$. Tal como se puede evidenciar en figura 2, la distribución de los subtipos moleculares fue significativamente diferente entre los grupos $(P<0,0000)$, ya que casos con niveles más altos de AR se clasifican, en su mayoría, como luminales B o HER2 enriquecidos (76,5\%), mientras que casos con niveles más altos de ER (AR/ER <2) preferencialmente se asignan al subtipo luminal A $(69,9 \%)$. Cabe resaltar que la distribución de subtipos en el grupo de casos ER- (242 casos) correspondió con lo esperado, ya que la mayor parte de estos se asignaron al subtipo molecular parecido al basal, ampliamente reconocidos por ser ER- y por su alta agresividad.

Figura 2. Distribución de subtipos moleculares en casos de CS. La asignación de subtipos moleculares fue significativamente diferente al comparar todos los grupos de estudio. Los casos ER+ se agruparon por la proporción AR/ER. Los valores al lado de cada barra indican el número de casos para cada subtipo molecular en los grupos de estudio

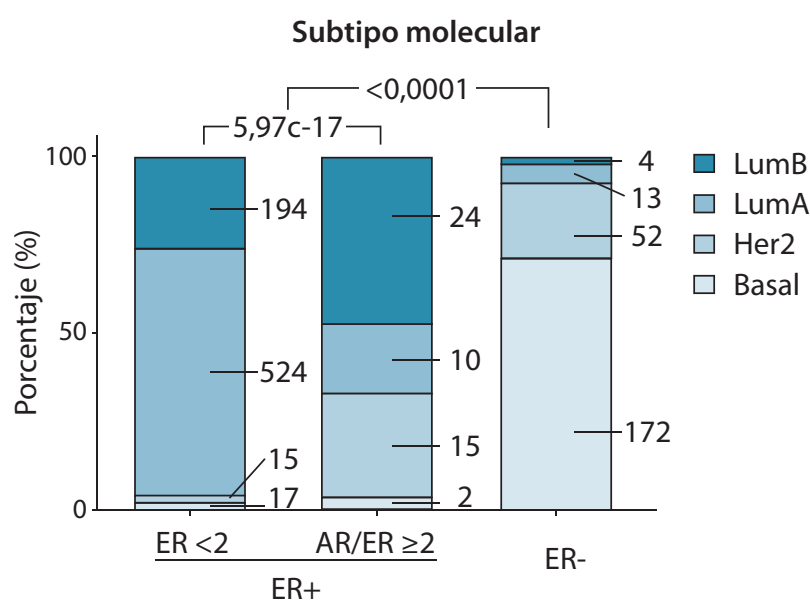

LumA: luminal A; lumB: luminal B; Basal: parecido al basal; ER-: caso receptor de estrógenos negativo; ER+: caso receptor de estrógenos positivo; HER2: HER2 enriquecido.

Valores de $p$ : prueba de comparaciones múltiples de Dunnet y Tuckey. 


\section{Discusión}

Comparado con el cáncer de próstata, el papel de AR en CS no es claro, ya que existen datos divergentes en cuanto al significado clínico y biológico de este receptor en carcinogénesis de mama. En CS ER+, la expresión de AR usualmente se ha asociado con mejores pronósticos, sin embargo, los resultados del presente estudio sugieren que al interior de casos luminales $(E R+)$ el subgrupo de tumores con proporción AR/ER $\geq 2$ tiene niveles de proliferación aumentados, lo cual confirma reportes recientes que demuestran peores pronósticos (períodos de supervivencia total y libre de la enfermedad más cortos) en casos de CS que presentan dicha característica ${ }^{(25,26)}$.

En línea con nuestros resultados se ha demostrado que la degradación/inhibición acelerada de AR, tanto in vivo como in vitro, promueve la detención del crecimiento y la apoptosis en células de CS MCF7 (ER+), siendo estos efectos bloqueados con la reintroducción de $\mathrm{AR}^{(25,32)}$. Asimismo, De Amicis y colaboradores estableció que tumores resistentes a tamoxifeno expresan altos niveles de AR y bajos niveles de ER, lo cual se asocia con niveles aumentados de proliferación celular y concuerda con resultados de estudios preclínicos, en los cuales se indica que casos con proporción $\mathrm{AR} / \mathrm{ER} \geq 2$ presentan riesgo incrementado de resistencia al tamoxifeno ${ }^{(25,33)}$. Adicionalmente, algunos reportes sugieren que AR está relacionado con invasividad y progresión del CS, dado que su expresión se ha detectado en un porcentaje significativamente alto de carcinomas in situ (CSI) adyacentes a carcinomas invasivos (CI) ${ }^{(34)}$. Además, aunque se ha demostrado que la expresión de receptores hormonales (ER/PgR) disminuye mientras que el CS progresa (de CIS a CI). Es notable que la expresión de AR se mantiene en un alto porcentaje de tumores metastásicos de $\operatorname{seno}^{(35,36)}$. Estas observaciones son soportadas por recientes estudios que demuestran que la expresión de AR tiene la habilidad de inducir la transición epitelio-mesenquimática y, por tanto, aumentan la capacidad migratoria e invasiva de células de $\mathrm{CS}^{(37)}$.

Por otro lado, los niveles aumentados en la SPC asociados con la proporción $A R / E R \geq 2$, junto con las características de agresividad conferidas a AR, concuerdan con la asignación de subtipos moleculares generados por el bioclasificador PAM50. De forma interesante, casos con mayores niveles de expresión de AR respecto a ER fueron preferencialmente asignados al subtipo luminal B $(47,1 \%)$. Dichos tumores son ampliamente caracterizados por tener altos niveles de expresión de marcadores de proliferación celular, así como peores pronósticos e incluso se ha sugerido que son entidades diferentes a los tumores luminales A, subtipo molecular, al cual se asignaron la mayoría $(\sim 70 \%)$ de casos de CS con proporción AR/ER<2 ${ }^{(38)}$.

Conforme a recientes publicaciones, nuestros análisis muestran que AR es también expresado en un porcentaje importante de tumores ER+ que sobreexpresan el marcador
HER2 ( 30\%), identificados como HER2 enriquecidos por el bioclasificador PAM50 ${ }^{(39,40)}$. En este subtipo molecular, que se caracteriza también por sus pobres pronósticos, se han identificado relaciones funcionales entre AR y HER2, que pueden potenciar la vía de señalización celular MAPK, implicada en la activación de la proliferación celular ${ }^{(40,41)}$. Además, Lin y colaboradores sugirieron que la sobreexpresión de HER2 se podría originar cuando los niveles de AR son más altos que los niveles de ER, lo cual concuerda con el elevado porcentaje de casos HER2 enriquecidos observados en el grupo AR/ER $\geq 2$ con respecto al grupo AR/ER $<2^{(22)}$.

Dado que la positividad de AR se ha relacionado con progresión del CS, nuestros datos sugieren que casos con proporción AR/ ER $\geq 2$ podrían ser tumores que están progresando a un estado más avanzado de la enfermedad, en donde altos niveles de AR y bajos niveles de ER confieren a dichos casos características biológicas de tumores más agresivos, lo que se evidencia no solo por mayores niveles de proliferación celular, sino también por la identificación preferencial de subtipos moleculares que usualmente presentan pobres pronósticos.

\section{Conclusión}

Los resultados de nuestro estudio muestran que altos niveles de expresión de AR pueden promover proliferación no solo de células de CS ER-, sino también de tumores luminales que presentan bajos niveles de expresión de ER; lo anterior teniendo en cuenta que en casos de $\mathrm{CS}$ con proporción $\mathrm{AR} / \mathrm{ER} \geq 2$. Se observó expresión aumentada de la SPC (AURKb, BUB1B, BUB1, CDK1 y CHEK1) con respecto a casos con proporción $\mathrm{AR} / \mathrm{ER}<2$. Adicionalmente, la clasificación por subtipos moleculares indica que casos con proporción $\mathrm{AR} / \mathrm{ER} \geq 2$ son principalmente asignados a subtipos con comportamiento biológico más agresivo y de peor pronóstico (luminar B y HER2 enriquecido). Tomados juntos, estos resultados sugieren la importancia de considerar la evaluación conjunta de los marcadores ER y AR en la práctica clínica de rutina para mejorar la clasificación de pacientes con CS de ER+y refuerzan la idea de utilizar inhibidores de AR para el tratamiento de pacientes con CS ER+ que presentan altos niveles de expresión del mismo.

\section{Agradecimientos}

Los autores agradecen a Isabella Castellano MD PhD y Laura Annaratone $\mathrm{PhD}$, profesoras/investigadoras de la Universidad de Turín, Italia, por su valiosa colaboración en la revisión crítica del presente documento.

\section{Conflicto de intereses}

Los autores declaran no tener ningún tipo de conflicto de interés. 


\section{Referencias}

1. Early Breast Cancer Trialists' Collaborative Group, Davies C, Godwin J, Gray R, Clarke M, Cutter D, et al. Relevance of breast cancer hormone receptors and other factors to the efficacy of adjuvant tamoxifen: patientlevel meta-analysis of randomised trials. Lancet. 2011;378(9793):771-84.

2. Hu R, Dawood S, Holmes M, Collins L, Schnitt S, Cole K, et al. Androgen receptor expression and breast cancer survival in postmenopausal women. Clin Cancer Res. 2011:17(7):1867-74.

3. Park S, Koo J, Park H, Kim J, Choi S, Lee J, et al. Expression of androgen receptors in primary breast cancer. Ann Oncol. 2010;21(3):488-92.

4. Aleskandarany M, Abduljabbar R, Ashankyty I, Elmouna A, Jerjees D, Ali S, et al. Prognostic significance of androgen receptor expression in invasive breast cancer: transcriptomic and protein expression analysis. Breast Cancer Res Treat. 2016;159(2):215-27.

5. Bozovic-Spasojevic I, Zardavas D, Brohee S, Ameye L, Fumagalli D, Ades F, et al. The prognostic role of androgen receptor in patients with early-stage breast cancer: a meta-analysis of clinical and gene expression data. Clin Cancer Res. 2017;23(11):2702-12.

6. Castellano I, Allia E, Accortanzo V, Vandone A, Chiusa L, Arisio R, et al. Androgen receptor expression is a significant prognostic factor in estrogen receptor positive breast cancers. Breast Cancer Res Treat. 2010;124(3):607-17.

7. Niemeier L, Dabbs J, Beriwal S, Striebel JM, Bhargava R. Androgen receptor in breast cancer: expression in estrogen receptor-positive tumors and in estrogen receptor-negative tumors with apocrine differentiation. Mod Pathol. 2010;23(2):205-12.

8. Asano Y, Kashiwagi S, Goto W, Tanaka S, Morisaki T, Takashima T, et al. Expression and clinical significance of androgen receptor in triple-negative breast cancer. Cancers (Basel). 2017:9(1).

9. Hilborn E, Gacic J, Fornander T, Nordenskjold B, Stal O, Jansson A. Androgen receptor expression predicts beneficial tamoxifen response in estrogen receptor-alpha-negative breast cancer. Br J Cancer. 2016;114(3):248-55.

10. Jiang H, Kuang X, Sun W, Xu Y, Zheng Y, Liu Y, et al. Androgen receptor expression predicts different clinical outcomes for breast cancer patients stratified by hormone receptor status. Oncotarget. 2016;7(27):41285-93.

11. Luo X, Shi Y, Li Z, Jiang W. Expression and clinical significance of androgen receptor in triple negative breast cancer. Chin J Cancer. 2010;29(6):585-90.

12. Pistelli M, Caramanti M, Biscotti T, Santinelli A, Pagliacci A, De Lisa M, et al. Androgen receptor expression in early triple-negative breast cancer: clinical significance and prognostic associations. Cancers (Basel). 2014;6(3):1351-62

13. Qu Q Mao Y, Fei X, Shen K. The impact of androgen receptor expression on breast cancer survival: a retrospective study and meta-analysis. PLoS One. 2013;8(12):e82650.

14. Vera-Badillo F, Templeton A, de Gouveia P, Diaz-Padilla I, Bedard P, AlMubarak M, et al. Androgen receptor expression and outcomes in early breast cancer: a systematic review and meta-analysis. J Natl Cancer Inst. 2014;106(1):djt319.

15. Peters A, Buchanan G, Ricciardelli C, Bianco-Miotto T, Centenera MM, Harris JM, et al. Androgen receptor inhibits estrogen receptor-alpha activity and is prognostic in breast cancer. Cancer Res. 2009;69(15):6131-40.

16. Cops E, Bianco-Miotto T, Moore N, Clarke C, Birrell S, Butler L, et al. Antiproliferative actions of the synthetic androgen, mibolerone, in breast cancer cells are mediated by both androgen and progesterone receptors. J Steroid Biochem Mol Biol. 2008;110(3-5):236-43.

17. Greeve M, Allan R, Harvey J, Bentel J. Inhibition of MCF-7 breast cancer cell proliferation by 5alpha-dihydrotestosterone; a role for p21(Cip1/Waf1). J Mol Endocrinol. 2004;32(3):793-810.

18. Macedo L, Guo Z, Tilghman S, Sabnis G, Qiu Y, Brodie A. Role of androgens on MCF-7 breast cancer cell growth and on the inhibitory effect of letrozole. Cancer Res. 2006;66(15):7775-82.

19. Need E, Selth L, Harris T, Birrell S, Tilley W, Buchanan G. Research resource: interplay between the genomic and transcriptional networks of androgen receptor and estrogen receptor alpha in luminal breast cancer cells. Mol Endocrinol. 2012;26(11):1941-52.

20. Buchanan G, Birrell S, Peters A, Bianco-Miotto T, Ramsay K, Cops E, et al. Decreased androgen receptor levels and receptor function in breast cancer contribute to the failure of response to medroxyprogesterone acetate. Cancer Res. 2005;65(18):8487-96.
21. Birrell S, Bentel J, Hickey T, Ricciardelli C, Weger M, Horsfall D, et al. Androgens induce divergent proliferative responses in human breast cancer cell lines. J Steroid Biochem Mol Biol. 1995;52(5):459-67.

22. Lin H, Sun M, Lin C, Tang H, London D, Shih A, et al. Androgen-induced hu man breast cancer cell proliferation is mediated by discrete mechanisms in estrogen receptor-alpha-positive and -negative breast cancer cells. Steroid Biochem Mol Biol. 2009;113(3-5):182-8.

23. Lehmann B, Bauer J, Chen X, Sanders M, Chakravarthy A, Shyr Y, et al Identification of human triple-negative breast cancer subtypes and preclinical models for selection of targeted therapies. J Clin Invest. 2011;121(7):2750-67.

24. Robinson J, Macarthur S, Ross-Innes C, Tilley W, Neal D, Mills I, et al. Androgen receptor driven transcription in molecular apocrine breast cancer is mediated by FoxA1. EMBO J. 2011;30(15):3019-27.

25. Cochrane D, Bernales S, Jacobsen B, Cittelly D, Howe E, D’Amato N, et al. Role of the androgen receptor in breast cancer and preclinical analysis of enzalutamide. Breast Cancer Res. 2014;16(1):R7.

26. Rangel N, Rondon-Lagos M, Annaratone L, Osella-Abate S, Metovic J, Mano $\mathrm{M}$, et al. The role of the AR/ER ratio in ER-positive breast cancer patients. Endocr Relat Cancer. 2018;25(3):163-72.

27. Iwamoto T, Booser D, Valero V, Murray I, Koenig K, Esteva F, et al. Estrogen receptor (ER) mRNA and ER-related gene expression in breast cancers that are $1 \%$ to $10 \%$ ER-positive by immunohistochemistry. J Clin Oncol. 2012;30(7):729-34

28. van 't Veer L, Dai H, van de Vijver M, He Y, Hart A, Mao M, et al. Gene expression profiling predicts clinical outcome of breast cancer. Nature. 2002;415(6871):530-6.

29. Parker J, Mullins M, Cheang M, Leung S, Voduc D, Vickery T, et al. Supervised risk predictor of breast cancer based on intrinsic subtypes. J Clin Oncol. 2009;27(8):1160-7.

30. Colaprico A, Silva TC, Olsen C, Garofano L, Cava C, Garolini D, et al. TCG Abiolinks: a R/Bioconductor package for integrative analysis of TCGA data. Nucleic Acids Res. 2016;44(8):e71.

31. Team RC. R: a language and environment for statistical computing Vienna, Austria: R Foundation for Statistical Computing; 2016. Disponible en: https://www.R-project.org/

32. Liao Y, Xia X, Liu N, Cai J, Guo Z, Li Y, et al. Growth arrest and apoptosis in duction in androgen receptor-positive human breast cancer cells by inhibition of USP14-mediated androgen receptor deubiquitination. Oncogene. 2018;37(14):1896-910.

33. De Amicis F, Thirugnansampanthan J, Cui Y, Selever J, Beyer A, Parra I, et al. Androgen receptor overexpression induces tamoxifen resistance in human breast cancer cells. Breast Cancer Res Treat. 2010;121(1):1-11.

34. Yu Q Niu Y, Liu N, Zhang J, Liu T, Zhang R, et al. Expression of androgen receptor in breast cancer and its significance as a prognostic factor. Ann Oncol. 2011;22(6):1288-94

35. Grogg A, Trippel M, Pfaltz K, Ladrach C, Droeser R, Cihoric N, et al. An drogen receptor status is highly conserved during tumor progression of breast cancer. BMC Cancer. 2015;15:872.

36. Hanley K, Wang J, Bourne P, Yang Q, Gao A, Lyman G, et al. Lack of expression of androgen receptor may play a critical role in transformation from in situ to invasive basal subtype of high-grade ductal carcinoma of the breast. Hum Pathol. 2008;39(3):386-92.

37. Feng J, Li L, Zhang N, Liu J, Zhang L, Gao H, et al. Androgen and AR contribute to breast cancer development and metastasis: an insight of mechanisms. Oncogene. 2017;36(20):2775-90.

38. Ades F, Zardavas D, Bozovic-Spasojevic I, Pugliano L, Fumagalli D, de Azambuja E, et al. Luminal B breast cancer: molecular characterization, clinical management, and future perspectives. J Clin Oncol. 2014;32(25):2794-803.

39. Al-Zahrani K, Cook D, Vanderhyden B, Sabourin L. Assessing the efficacy of androgen receptor and Sox10 as independent markers of the triplenegative breast cancer subtype by transcriptome profiling. Oncotarget. 2018;9(70):33348-59.

40. Daemen A, Manning G. HER2 is not a cancer subtype but rather a pancancer event and is highly enriched in AR-driven breast tumors. Breast Cancer Res. 2018;20(1):8.

41. Chia K, Liu J, Francis G, Naderi A. A feedback loop between androgen receptor and ERK signaling in estrogen receptor-negative breast cancer Neoplasia. 2011;13(2):154-66. 